\title{
MICROBIOME
}

\section{Of microbiome and metabolome in the bobtail squid}

Koch, E.J. et al. J Exp Biol 223, jeb212860 (2020) https://doi.org/10.1242/jeb.212860

The Hawaiian bobtail squid, Euprymna scolopes, and the bacterium Vibrio fischeri have a unique relationship: it's one to one. The bioluminescent bacterium is the sole microbial inhabitant of the bobtail squid's light organ. The bacterium gets a safe haven and in return, it gives its host the ability to glow, which the squid takes advantage of to mimic moonlight through the water. This eliminates the night-time shadow that would otherwise give its presence away to its any predators below, leaving the squid to forage in peace.

In the lab, it's a tractable relationship: the squid can be cultured with or without Vibrio fisheri. Abiotic squid can then be colonized with the bacterium at any point during their life cycle. This affords researchers the opportunity to study how a microbe influences its host, without having to tease apart competing contributions from other parties that may be present - a problem that complicates microbiome studies in animals with more diverse microbial assemblages.

A new study looks at the impact of the bobtail squid's simple microbiome on its metabolome, the sum of the small molecular metabolites that are the stuff of biochemical reactions in the body. The squid has a closed circulatory system, so the team sampled metabolites in its hemolymph - a fluid equivalent to vertebrate blood. Using gas chromatography mass spectromy analysis, they found 145 metabolites, which they classified into seven biochemical categories.

The relative abundance of the metabolites varied, and, sampled over three times points in a 24-hour period, reflected the squid's diel rhythms - every morning, the squid expel most of their symbionts, along with some of the epithelial tissue on which the bacterium grow, leaving those Vibrio that remain to recolonize the light organ before nightfall.

A number of those metabolites were significantly influenced by the presence of Vibrio, with differences in abundance observed among 35 metabolites in males and 27 metabolites in females between those with and without their glowing partner. In males, however, most of those metabolites increased in abundance in symbiotic animals; the reverse was true in females, suggesting that there is also a sexually dimorphic element to the squid's symbiotic relationship.

"By applying metabolomics to the squid-vibrio symbiosis, this study shows how a natural colonization can not only affect the symbiotic organ, but also change overall host biochemistry by influencing daily rhythms within the metabolome," the authors conclude.

\section{Ellen P. Neff}

Published online: 12 October 2020

https://doi.org/10.1038/s41684-020-00670-2

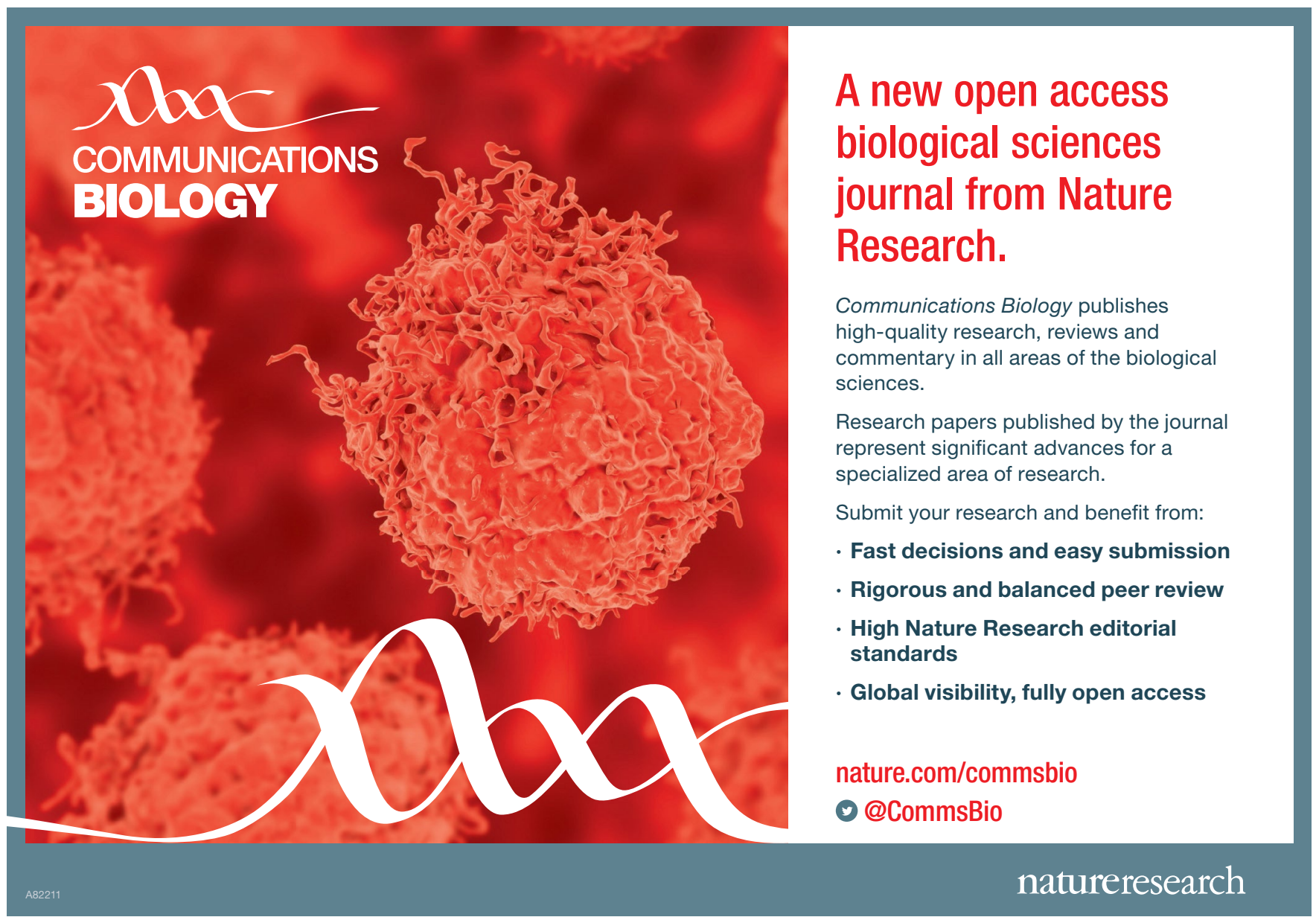

